\title{
Lessons learned from Community- based Management of Acute Malnutrition (CMAM) programmes that operate in fragile or conflict affected settings
}

\author{
Evert-jan Quak \\ Institute of Development Studies (IDS) \\ 8 October 2021
}

\section{Question}

What are the lessons learned from CMAM programmes in fragile or conflict affected settings? When possible, please focus on lessons learned and impacts associated with community data tracking and simplified nutrition surveillance processes (e.g. Family MUAC - Mid-Upper Arm Circumference).

\section{Contents}
1. Summary
2. What is CMAM
3. General lessons learned from CMAM evaluations
4. Lessons learned from conflict settings
5. Appendix: Logic model used for CMAM evaluations
6. References

\footnotetext{
The K4D helpdesk service provides brief summaries of current research, evidence, and lessons learned. Helpdesk reports are not rigorous or systematic reviews; they are intended to provide an introduction to the most important evidence related to a research question. They draw on a rapid deskbased review of published literature and consultation with subject specialists.
} 


\section{Summary}

This rapid review synthesises the literature from academic, policy, and knowledge institution sources on the lessons learned on how community-based management of acute malnutrition (CMAM) programmes could be adapted in settings of conflict and fragility. Most of the academic CMAM literature focus on conceptualising and measuring issues of malnutrition and some on the cost effectiveness of these programmes. The lessons learned are more commonly mentioned in the 'grey literature' from international humanitarian agencies and non-governmental organisations (NGOs). Most of the literature does not make a distinction between conflict and non-conflict settings. Having said that, some interesting programmes and literature were found that looked at the feasibility of simplified or adapted protocols for CMAM in conflict and fragile settings, mostly in South Sudan and Yemen. Since some lessons from CMAM programmes in non-conflict settings are of interest for programmes in conflict settings, the report has included them in section 3 .

The literature is clear that quality and effectiveness of CMAM services depend on the health system (e.g. sustained financing, competent and responsive workforce, consistent stock of equipment), community engagement (e.g. to mobilise, screen, refer and follow up), and linkages with other programmes (e.g. nutrition education and counselling; water, sanitation, hygiene (WASH); and early childhood development).

- Integration in health systems: The preferred way is to have community health volunteers (CHVs) operating in close cooperation with professional health workers based in government run health centres. However, there is acknowledgement that in cases where that is not possible, due to a lack of governmental structures or in emergency situations, established health NGOs can fill the gap by providing stand-alone treatment services, but for a limited duration and with support from provincial and/or district authorities or leaders. Preferably, the aim should be to integrate the CMAM approaches within the health system at some point. A study based on experiences in Bangladesh shows that such integration is not easy and needs preparation, coordination, and time. Health staff were short of knowledge on facility-based management of children with severe acute malnutrition (SAM) referred to the centre by $\mathrm{CHVs}$, while supplies of medicines and therapeutic diets were often interrupted.

- Community engagement: Community mobilisation increases when programmes include community sensitisation activities, including traditional and religious leaders, over a longer period of time. Assigning focal persons for community mobilisation at all levels between district and villages is recommended and programmes should seriously consider community participation in decision-making processes. Giving communities more ownership of CMAM programmes increases support in the community, which relates to higher number of children screened and treated for malnutrition. Regular meetings of community health groups/committees should take place, while programme staff collect feedback during regular visits to villages.

- Integrating with other programmes: Studies show the importance of linking CMAM with other community-based programmes that aim to increase access to safe water and sanitation facilities; health and nutrition services; improved agricultural production and diversification of livelihoods for the extreme poor; development of early warning systems; strengthened community organisations; and the increased participation of women in 
community organisations. Prevention should move more to the forefront in acute malnutrition programmes, but (for good reasons) due to limited funding CMAM often focuses on the SAM cases, while integrating moderate cases of malnutrition in the programme would be beneficial to avoid cases to become severe.

Family MUAC (Mid-Upper Arm Circumference) is used to increase community participation and detect early cases more effectively. Studies show that MUAC provided by caregivers (often mothers) were much less likely to require inpatient care. Although training caregivers requires higher up-front costs, overall costs spread over time become much lower due to early detection of moderate acute malnutrition (MAM) and SAM without complications. However, refresher trainings are important as MUAC outcomes depend on the skill of the user, which will deteriorate after some time. It is also important in Family MUAC projects to include CHVs and communicate well with them about the benefits for their work of Family MUAC as they might be afraid to lose some of their work.

The CMAM literature does not say a lot about $\mathrm{m}$-Health solutions (using mobile devises and applications) in data collection and surveillance systems. Some studies have been identified that show the benefits of $\mathrm{m}$-Health in coordination with $\mathrm{CHVs}$ (e.g. India and Nigeria), but no information could be found how this could be extended to caregivers in community (e.g. Family MUAC, nutrition education).

Many of the above-mentioned issues are relevant for CMAM programmes in settings of nonemergency, emergency, conflict and fragility. However, there are special circumstance (lack of health facilities and staff, restrained coordination, broken supply chains) in conflict and fragile settings that need adaptation and simplification of the standard protocols. The literature often speaks about combined (combining SAM and MAM) and simplified protocols (e.g. lower frequency of follow-up meetings and supplies). Adaptations also mentioned in the literature is the use of mobile health centres and (or in combination) implementation of a Rapid Response Mechanism (RRM) within CMAM.

Because of a broken or partly broken health system (e.g. supplies and motivated staff) in settings of conflict and fragility, this also means that local governments are not able to fund access for many people to adequate inpatient and outpatient treatment centres. NGOs and humanitarian agencies are often able to set up stand-alone OTPs or mobile centres in the most affected regions. Training CHVs is important and implementing Family MUAC to organise, mobilise, and engage with local communities. Importantly, research shows that:

- Low literacy of CHVs is not a problem to achieve good nutritional outcomes as long as protocols are simplified.

- Combined/simplified protocols are not inferior to standard protocols. However, due to complexities and low funding, treatment is focused on SAM and availability for children with MAM is far less prioritised, until they deteriorate to SAM.

- There is widespread confusion about combined/simplified protocol terminology and content, because there is no coherence at the global level. This results in ad hoc approaches. 


\section{What is CMAM}

In 2001, Community-based Management of Acute Malnutrition (CMAM) evolved from Community-based Therapeutic Care (CTC) approaches with the aim to manage (e.g. measure and treat) acute malnutrition as much as possible within communities (FANTA, 2018; UNICEF, 2013). The approach was endorsed by UN agencies in 2007 (WHO et al., 2007). Although the term malnutrition refers to both under-nutrition and over-nutrition, in the context of CMAM it generally indicates under-nutrition including protein-energy malnutrition and deficiency of micronutrients (Park et al., 2012). In some countries (e.g. Kenya), CMAM is referred to as the integrated management of acute malnutrition (IMAM) (UNICEF, 2013).

The 2013 Lancet Series on Maternal and Child Undernutrition identified 10 high impact, highly cost-effective nutrition interventions. CMAM was estimated to have the greatest impact on underfive deaths $(435,000$ lives saved per year globally) at the lowest cost per life saved (US\$125) (Bhutta et al., 2013). This is in line with research findings, often at programme or country level, that show the cost-efficiency of CMAM approaches (e.g. Aguayo et al., 2018, for Pakistan; Goudet et al., 2018, for Mumbai slum programme; Wilunda et al., 2021, for Tanzania).

The CMAM approach consists of four main components (Park et al., 2012; UNICEF, 2013; FANTA, 2018). The start point is community outreach and mobilisation because the success of a CMAM programme relies on early case finding combined with effective follow up actions at the community level. This means that community health volunteers ( $\mathrm{CHVs}$ ) need to be selected and trained to identify malnourished children. Depending on the severity of malnutrition each identified child falls into one of three treatment regimens, also referred to as 'triage approach':

- Inpatient treatment in Stabilisation Centres or Therapeutic Feeding Centres (24 hours care between 2-3 weeks) for the management of severe acute malnutrition (SAM) cases with medical complications.

- Outpatient care for the management of SAM without medical complications at home with weekly or biweekly visits at a nearby health facility within an Outpatient Therapeutic Programme (OTP).

- Treatment of moderate acute malnutrition (MAM) cases is part of a Supplementary Feeding Programme (SFP).

CMAM programmes have developed strict but easy-to-use measurement protocols by voluntary health workers of a child's level of malnutrition. They check on three levels: presence of bilateral pitting oedema, Mid-Upper Arm Circumference (MUAC), and Weight for Height (WFH) index (FANTA, 2018). Complications that accompany SAM are often respiratory infections, severe dehydration (which could be combined with diarrhoea), low blood sugar levels (hypoglycaemia), and a low body temperature (hypothermia) (Park et al., 2012). See Table 1 and Figure 1 for specific malnutrition criteria. 
Table 1. Acute malnutrition measures for children aged 6-59 months (Source: FANTA, 2018)

\begin{tabular}{|l|l|l|l|}
\hline & Bilateral Pitting Oedema & MUAC & $\begin{array}{l}\text { WFH z-score (WHO } \\
\text { standards) }\end{array}$ \\
\hline SAM & Present & $<115 \mathrm{~mm}$ & $<-3$ \\
\hline MAM & Not present & $>115 \mathrm{~mm}$ and $<125 \mathrm{~mm}$ & $>-3$ and $<-2$ \\
\hline
\end{tabular}

Figure 1. Three pathways of treatment of infants (6-59 months) for acute malnutrition Source: Park et al., 2012 CC-BY-NC

The training guide for trainers of CMAM published by the Food and Nutrition Technical Assistance III Project (FANTA) mentions in their 2018 revised and updated version that they prefer the term 'nutrition vulnerability' instead of 'acute malnutrition' for infants under 6 months old, because it captures broader characteristics of the needs of these infants that also addresses underlying problems such as ability to feed, underlying medical conditions, maternal illness and poor mental health, and adverse social circumstances (FANTA, 2018: iv). For infants between 659 months of age the guide still refers to 'acute malnutrition'. World Vision, who started using CMAM in Niger in 2006, states that CMAM should be implemented in any area where at least $10 \%$ of children under 5 are moderately malnourished (low weight for height) and there are aggravating factors present, such as generalised food insecurity, widespread communicable diseases and high crude death rate. ${ }^{1}$

CMAM was originally designed for the emergency context, as an alternative to the traditional model of rehabilitating all severely malnourished children through in-patient care at Therapeutic Feeding Centres but is now also often used for long-term development programming. ${ }^{2}$ The CMAM literature makes the argument for these programmes in emergency and non-emergency settings (WHO et al., 2007; UNICEF, 2013; Park et al., 2012):

- CMAM is a cost-efficient approach to identify, treat, and monitor large amounts of children with malnutrition in a timely manner, which is vital within emergency famine situations.

- The approach is also efficient in non-emergency situations in Low- and Middle-Income Countries (LMICs) where the malnutrition inpatient care model is not sustainable because of poor infrastructure and lack of skilled professional health workers.

\footnotetext{
${ }^{1}$ Information retrieved from the website of World Vision: https://www.wvi.org/nutrition/project-models/cmam

${ }^{2}$ Information retrieved from the website of World Vision: https://www.wvi.org/nutrition/project-models/cmam
} 
The literature states that CMAM in non-emergency settings should preferably take place in ongoing health programming with inpatient care at existing health facilities with 24-hour care (e.g., hospitals, health centres with hospitalisation) and outpatient care operates at the first-level health facility (e.g., clinics, health posts) (WHO, 2007; UNICEF, 2013). According to a 2012 Global CMAM Synthesis Survey most CMAM interventions are either non-emergency programmes being integrated into health system functions, or a combination of non-emergency and emergency, with relatively few purely emergency responses (UNICEF, 2013). In most countries, CMAM is managed by the government, with support from NGO implementing partners, UNICEF, WHO, and WFP, and a host of other partners (UNICEF, 2013).

A model based purely on hospital-based management of malnutrition not only is costly and resource-intensive as inpatient care demands many qualified and trained staff, plus a vast number of inpatient beds, but in the context of LMICs such approaches often have low coverage rates (which is worsened in situations where dispersed communities live in chronic poverty), while overcrowded with potential threats for cross-infection (Park et al., 2012; USAID, 2016). Treatment facilities are often far away from communities and can last for weeks for SAM which is a substantial burden and cost of illness for caregivers (USAID, 2016).

Children identified with SAM and medical complications (often combined with no appetite) still need to be treated with inpatient care programmes. With community engagement and mobilisation and screening for malnutrition in communities, children with malnutrition can be identified early on which has the potential to reduce the number of children in need of inpatient care. ${ }^{3}$ However, this will only happen when community outreach is effective and community interventions, such as supplementary feeding, timely (UNICEF, 2013).

The treatment in communities of children identified with malnutrition has become possible because of the innovation of ready-to-use therapeutic food (RUTF) that can be produced and distributed locally (WHO, 2007; Park et al., 2012). ${ }^{4}$ Thorough inspections and quality control are needed for local production to ensure that there is no risk of contamination of the ingredients and that the product has the right composition and quality (FANTA, 2018).

See in Appendix 1 the model used in many CMAM programmes explaining inputs, activities, outputs, outcomes and impacts. The guiding principles that should underpin all CMAM programmes as mentioned in Box 1 relate to this CMAM logic model.

\section{Box 1: The four guiding principles of CMAM programmes}

Maximum coverage and access. Community mobilisation is pivotal as it is the only way to secure the greatest coverage by securing maximum access to populations in need (which can be rural communities or slum dwellers in urban areas).

\footnotetext{
${ }^{3}$ Once stabilised, hospitalised children with SAM are referred to the Outpatient Therapeutic Programme (OTP) clinic to continue the SAM treatment course within the community.

${ }^{4}$ RUTF is an oil-based energy-dense paste with very low or no water content. It does not grow bacteria even when accidentally contaminated. It can be kept in simple packaging for several months without refrigeration. RUTF can be eaten straight from the packet or pot and can be consumed easily by children from the age of 6 months. Also, RUTF contains iron, vitamin A, and several other micronutrients needed for the child to recover (FANTA, 2018).
} 
Timeliness. The point is to identify malnourished cases in earlier stages before they become severe and reach complications. If there are any early warning signs of famine, the case finding activities should be strengthened to prevent escalation of malnutrition cases at communities.

Appropriate malnutrition care. CMAM should be simple and effective both for severely malnourished children with or without complication, with effective links between outpatient and inpatient care respectively.

Care for as long as it is needed. CMAM should provide care until the malnourished children recover and gain more than $15 \%$ of body weight. As long as there are acutely malnourished children in the targeted communities or indications of food and nutrition insecurity, the programme should be sustained.

Source: Park et al. (2012) CC-BY-NC

\section{General lessons learned from CMAM evaluations}

\section{Opportunities and challenges of CMAM programmes}

CMAM programmes enrolled in LMICs need time to be fully implemented and scaled up, and at times services can be disrupted. In particular the process to integrate CMAM within health systems is challenging (UNICEF, 2013). As such, what happens is that NGOs often fill in gaps. Health system capacity and the cost of specialised nutrition products are major challenges with full integration into national health systems (USAID, 2016).

The USAID Technical Guidance Brief (2016) states that the quality and effectiveness of CMAM services depend on the health system (e.g. sustained financing, competent and responsive workforce, consistent stock of equipment), community engagement (e.g. mobilise, screen, refer and follow up), and linkages with other programmes (e.g. nutrition education and counselling, WASH, early childhood development). On the supply side, people need to have physical access to well run, quality treatment centres, while on the demand side they need to be motivated enough to go and continue to go to subsequent visits and follow the regimen at home.

Programming should also be sensitive to gender, ensuring that roles and responsibilities for managing and preventing malnutrition are encouraged equally for men and women. UNICEF recommends that inter-sectoral links initially be tested and documented on a small scale to assess whether they do, in fact, benefit each other and result in more efficient use of resources (UNICEF, 2015).

To increase accessibility of the services and mobilise communities, The State of Acute Malnutrition website, has collected some practical tips on running CMAM programmes for practitioners. ${ }^{5}$ For example, to increase accessibility for more remote villages with less advanced integrated community health services (which could be temporary in initial stages of the CMAM programme), mobile clinics for OTPs and SFPs could be considered. Another option is to map potential partners and community members who could assist the local population with the provision of collective transport to the nearest OTP site and approach partners and community members to propose coordination of transport. It could also be considered to reduce

${ }^{5}$ Information retrieved from The State of Acute Malnutrition website: https://acutemalnutrition.org/en/faq 
frequency of appointments at CMAM programmes to reduce the constraints of these travels for the community and keep them engaged (e.g. from weekly to fortnightly or monthly).

On the issue of how communities can be better sensitised for the CMAM programme, The State of Acute Malnutrition website recommends regular community outreach activities, such as monthly meetings with community leaders, CHVs and community groups (e.g. in health coordination meetings, village health committees), are important; as well as engaging with district or community media outlets (e.g. radio broadcasts). Assigning focal persons for community mobilisation at all levels between district and villages. To collect feedback and understand progress and challenges programme staff need to visit these villages and meet community groups and leaders on a regular base.

The State of Acute Malnutrition website also mentions that it is important to ensure genuine community participation and shared decision-making in CMAM programmes. To deliver on that the programme needs to support commitment and ownership of community actors in the CMAM programme by communicating clear roles and responsibilities for all community actors, while developing shared decision-making processes that are inclusive and guarantee that community members are involved.

The evidence from CMAM programmes shows clearly that the preferred way is to have CHVs that operate in close cooperation with the professional health workers in OTPs and inpatient treatment centres, which preferably are government run as part of the health system (Concern, 2015). In cases where that is not possible due to a lack of governmental structures, or in emergency situations, established health NGOs can fill the gap by providing stand-alone treatment services but for a limited duration and with support from provincial and district authorities (Concern, 2015).

The aim should be to integrate the CMAM approaches within the health system at some point and measures should be taken in advance to let that happen. This is important because, NGOs set up systems independently and at the end of their funding periods they found themselves trying to 'handover' a resource-intensive emergency programme to ill-equipped government health systems. This transition needs different skills from NGO staff (good communication and diplomacy skills, conduct joint planning and review) as the programme moves away from direct implementation in an emergency setting to supporting the Ministry of Health to integrate CMAM in the health system. As such, Concern (2015) states that there are some opportunities for existing health and community systems to be leveraged to achieve significant scale and impact of CMAM (see Box 2). ${ }^{6}$

Indeed, evaluations and research (see also case studies below) show the challenges involved in integrating CMAM within health systems. For example, Ireen et al. (2018) show this for Bangladesh where there is an enabling environment for CMAM intervention, although the current health system is not yet prepared for it. One issue raised is that 288 inpatient treatment facilities are operational across the country, only $2.7 \%$ doctors and $3.3 \%$ auxiliary staff are trained on facility-based management of SAM. Supplies of medicines and therapeutic diet to OTPS and

\footnotetext{
${ }^{6}$ Concern uses a CMAM surge approach in countries that are vulnerable for high food insecurity situations (e.g. in specific seasons) that integrates more surveillance and monitoring and capacity building within the system. See for more information https://www.concern.net/insights/cmam-surge-approach
} 
$\mathrm{CHVs}$ are often interrupted. Disagreement among nutrition stakeholders regarding the production specifications and protocols of RUTF is just one underlying problem. Nutrition coordination remains fragile because there is no functional supra-ministerial coordination platform for multisectoral and multi-stakeholder nutrition in Bangladesh (Ireen et al., 2018).

Box 2. Key steps for integration of CMAM into the health service:

- Ensure that all relevant country policies and operational strategies include CMAM as an essential (ideally free) child health service.

- Include RUTF on the essential drugs list. Forecasting for essential drugs must include the drugs used routinely in CMAM programmes.

- Conduct specific training to build MoH capacity in basic supply and logistics management, including transport and storage of ready-to-use therapeutic food (RUTF).

- Support countries to develop national costed nutrition plans.

- Conduct frequent in-service training on CMAM for all relevant health workers and include a CMAM module in health worker basic training curricula.

- Include CMAM responsibilities in the job descriptions of all relevant staff.

- Simplify and align individual SAM patient monitoring forms with existing case management forms and registers and the national Health Information Management System (HIMS).

- Include a limited number of observable CMAM assessment points in health service supervision checklists for health facility and community-level supervision.

- Integrate CMAM into Community Case Management where it exists.

\section{Source: Source: (c) Concern Worldwide 2015}

Linking acute malnutrition programmes with longer-term programmes for building resilience in communities and behaviour change in nutrition is also often mentioned as an important pillar for successful CMAM programmes. These include increasing access to safe water and sanitation facilities, increasing access to and use of high-quality health and nutrition services, improved agricultural production and diversification of livelihoods for the extreme poor, development of early warning systems, strengthened community organisations and the increased participation of women (Concern, 2015). There is also evidence that linking CMAM with social protection schemes are a useful way to reduce acute malnutrition in the lean season (e.g. in Niger see: Bliss et al., 2018) and improve diverse and healthier diets (e.g. in Ethiopia see: Fox, 2012).

Huybregts et al. $(2017,2019)$ show the importance of linking CMAM (acute malnutrition) with prevention interventions, which are important to increase community engagement and participation in screening and uptake and adherence to treatment. They show results from the $\mathrm{PROMIS}^{7}$ programme in Burkina Faso, Mali and Senegal that show significant increase of screening coverage due to introducing preventive interventions into acute malnutrition screening. The preventive interventions included provision of dietary supplements, providing essential nutrients and through behaviour change communication on nutrition, health, and hygiene

7 PROMIS stands for Innovative Approaches for the Prevention of Childhood Malnutrition and the IFPRI funded programme was implemented by Helen Keller International in Mali, Burkina Faso and Senegal between 20142017. 
practices. Including the nutritional supplement was expected to serve as an incentive for caregivers to bring their children to acute malnutrition screening sessions.

The findings show that using CMAM as a community platform for prevention interventions is effective at addressing important barriers to caregivers' participation in screenings due to distance and opportunity costs to travel to health centres, which in the long term will benefit CMAM programmes. However, Huybregts et al. $(2017,2019)$ also state that challenges remain that the use of $\mathrm{CHVs}$ might compromise on high-quality services and adherence to programme implementation guidelines.

\section{Integrate Family MUAC within CMAM}

The Family MUAC approach trains caregivers (mostly mothers) to identify early signs of malnutrition in their children using a simple to use Mid-Upper Arm Circumference (MUAC) tape. By moving this task to family members, they are empowered to manage their children's health and CHVs have more time to carry out other tasks, such as treatment of MAM and SAM without complications. ${ }^{8}$ The Covid-19 pandemic which limited mobility and face-to-face contacts showed the importance of training caregivers to screen their children on malnutrition (Save the Children, 2020; World Vision, 2020) and Family MUAC now has been promoted in the recently developed Global guidelines for nutrition service delivery in the context of COVID-19 (UNICEF et al. 2020).

MUAC is a simple screening tool that has been shown to be effective as a predictor of mortality in acutely malnourished children. Respondents to the 2012 Global CMAM Synthesis Survey selected stronger case identification through screening with MUAC and referral as the most important mechanism for increasing access and promoting equity (UNICEF, 2013). A more recent review on MUAC as a tool to detect malnutrition effectively (either by caregivers as CHVs) finds that caregivers can successfully screen MUAC (although they highlighted the small number of published research studies, their narrow geographic scope, and most described intensive, small-scale interventions) (Bliss et al., 2018). ${ }^{9}$

A study in rural Niger compared outcomes of MUAC provided by mothers and CHVs (Alé et al., 2016). The findings show that there was a higher rate of earlier detection of malnourished cases by Family MUAC. Children that were screened by their mothers were much less likely to require inpatient care, both at admission and during treatment, with the most pronounced difference at admission for those enrolled by MUAC. Although training mothers requires higher up-front costs, overall costs spread over time become much lower due to early detection (US $\$ 8,600$ vs US\$21,980) (Alé et al., 2016). Other studies show the same outcomes (e.g. Blackwell et al.,

\footnotetext{
8 Information retrieved from The State of Acute Malnutrition website https://www.acutemalnutrition.org/en/FamilyMUAC

${ }^{9}$ Other studies agree as in most populations, children identified as severely wasted by MUAC will generally be at higher risk of death than those identified by WFH/WHZ (Briend et al., 2012). Therefore, it has been recommended that where resources are limited, it is preferable to screen by MUAC than by WHZ (Myatt, Khara, \& Collins, 2006). However, some researchers have alerted that a significant number of children in need could be missed when MUAC is used as the only admission criteria and recommend that both MUAC and WHZ be used (Grellety and Golden, 2016).
} 
2015). For more detailed information about evidence from peer reviewed articles on Family MUAC see the rapid review of UNICEF (2020).

Trainings and refresher trainings are important as MUAC outcomes depend on the skill of the user, particularly regarding the tension placed on the tape, and its interpretation (UNICEF, 2013). In Afghanistan, Action for Development, developed a project to train CHVs in Family MUAC in five provinces. In this case the project offered a cash incentive per volunteer to be paid on completion of trainings. Giving caregivers the opportunity to screen their children was important due to the security situation combined with the Covid-19 outbreak that limits travels to health centres and by $\mathrm{CHVs}{ }^{10}$

Shared lessons from guidance ${ }^{11}$ on Family MUAC show that it is important to make health centre staff aware of Family MUAC and take away their concerns of mothers having MUAC tapes. Some advocacy is needed to ensure health staff (including CHVs) to understand how Family MUAC can make their work easier rather than taking away aspects of their work. They would become more trainers and manage caretakers' screening activities. A World Vision project in Mauritania mentions that they included grandparents in the training to increase positive outcomes. ${ }^{12}$ Trainings often include other signs of malnutrition such as bilateral pitting oedema. Furthermore, newly trained family members could conduct an initial mass screening in villages under supervision of $\mathrm{CHVs}$.

Importantly, caregivers should know who to contact after the detected signs of malnutrition. In situations where they live far from health centres, they should take their child immediately to the $\mathrm{CHVs}$ for further screening. A large supply of MUAC tapes should be planned and in order not to lose the MUAC tapes, a string should be put on the tape to hang up in the house which also could serve as a further reminder to screen their children on a regular basis. One critical challenge is the lack of reporting tools to capture Family MUAC screening separately from MUAC screening from $\mathrm{CHVs}$.

\section{Incorporating m-Health}

There are few experiences of using $\mathrm{m}$-Health tools in the screening processes of $\mathrm{CHVs}$ (see case study on Nigeria which mentions the use of SMS in a CMAM programme's information system). No information could be found on m-Health tools to support Family MUAC, but the World Vision Health and Nutrition Technical Brief to adapt CMAM programmes to Covid-19 realities makes some references in using ICTs, particularly mobile phones (e.g. through SMS), in collecting data and maintaining databases, and continuing trainings (World Vision, 2020).

One study shows the opportunities of using m-Health in CMAM, based on a project in Mumbai (Chanani et al., 2016). CHVs collect monitoring data to track malnutrition status by using Android smartphones and a mobile application (CommCare). The app is able to compile longitudinal data

\footnotetext{
10 This information was retrieved from The State of Acute Malnutrition website https://acutemalnutrition.org/en/SUN-Afghanistan

11 See ALIMA (2016) and World Vision (2017).

12 This information was retrieved from The State of Acute Malnutrition website https://acutemalnutrition.org/en/World-Vision
} 
records for each person and to aid the CMAM programme in prioritising and monitoring the health status of children and pregnant women. However, this is only possible due to high network coverage in a city as Mumbai. When coverage improves in rural setting this might become useful in some countries in the near future. Chanani et al. (2016) conclude that the use of mobile phones to collect basic data and adhere to treatment protocols was successfully adopted by the $\mathrm{CHVs}$ and reduced mistakes. To even further minimise errors after the pilot phase in 2013 an automated calculation of nutritional grades was added. This strategy significantly reduced the error rate in assessing acute malnutrition at the field level by approximately $5 \%$. The mobile calculator also reduces the variability in error rates across community health workers.

\section{Case studies}

\section{Zambia $^{13}$}

The Rainbow Project in Zambia combines the treatment and prevention of MAM and SAM and underweight through SFPs. Since 2001, the focus of the project has been on establishing 11 SFPs in the Ndola district where it operates and run by small, local NGOs and community-based organisations and coordinated and supported by the Rainbow Project office (Moramarco et al., 2019). This office works in close collaboration with local health facilities, the district children's hospital, the Ndola District Health Management Teams (DHMTs) and other health authorities. Weekly activities at each SFP include anthropometric assessment and monitoring; cooking demonstrations; group health talk sessions; individual nutrition counselling; on-site meal provision and distribution of take-home high-energy protein supplement (HEPS) and local food.

In 2008, six OTPs were established for the management of SAM, in collaboration with the DHMT and in 2011 all OTPs were handed over to the district authorities. However, SFPs remain the frontline provision of care within the communities even enrolling routinely SAM cases (without complications) as there were many challenges within OTPs, such as supply shortages of RUTF and challenges associated with integration into routine services at the local clinics where they are co-located. As RUTF were frequently undersupplied, SAM children received extra HEPS and local food such as maize flour, oil, sugar, and groundnuts were given to recipient family. Having said that, OTP remained the first choice for uncomplicated SAM treatment, but by accommodating SAM children in the SFP helped to compensate for shortfalls in OTP services (Moramarco et al., 2018, 2019).

Research and evaluations by Moramarco et al. $(2017,2018,2019)$ of this CMAM programme in Zambia identified weaknesses at the start, such as poor written planning, lack of documentation of the activities, lack of supervision and poor individual nutrition counselling. Adaptations were made to the programme in response, including the development of a printed register to standardise documentation across the SFPs, enhanced centre supervision, and improve quality of nutrition counselling. As the findings showed that nutrition counselling could not be conducted effectively by $\mathrm{CHVs}$ alone at that stage, since 2015 a local nutritionist has been employed by the programme to better target counselling quality and effectiveness. Follow-up research has shown that this recognition of nutrition-sensitive and nutrition-specific activities in the SFP has allowed mothers to acquire basic nutrition skills and child health knowledge. On a specific note, the

\footnotetext{
${ }^{13}$ Sources for this case study are: Moramarco et al. $(2017,2018,2019)$.
} 
Rainbow Project also demonstrates that in regions with high burdens of HIV infections, special attention should be given to integrate prevention and treatment of malnutrition with HIV programmes.

\section{Angola ${ }^{14}$}

In four provinces (Huambo, Bie, Kwanza Sul, Zaire) a CMAM programme was established in response to a drought that impacted 2011/2012 harvest. At that time there was little nutrition capacity in the region with most nutrition NGOs closed in 2007. In 2012, three NGOs (World Vision, Africare, People in Need) started the programme in cooperation with UNICEF and the provincial and municipal health departments. The focus of the three NGOs was on community mobilisation and training CHVs that provide treatment of uncomplicated SAM and MAM children. The programme linked with government run health facilities that provide inpatient and outpatient care (OTPs) within established health centres for treatment of complicated cases.

The programme relies on a network of 2,044 CHVs (Emary, 2021). Community mobilisation was organised through "Sobas" (traditional leaders) to promote better feeding practices and CMAM activities. Working with UNICEF, provincial and municipal health departments ensured the delivery of RUTF and coordination of referrals from villages in OTPs and inpatient care. The programme linked with existing programmes for feeding practices for mothers with malnourished children - water treatment, food diversification, exclusive breastfeeding.

Selection criteria for $\mathrm{CHV}$ included literacy, respected by community, and knowledge of health. Many had past experience in community health programmes and were identified by Sobas and village leaders. The training was provided by the NGOs by using UNICEF materials. Each group of activists is assigned to one commune and is coordinated by the Commune Supervisor, who ensures that they cover all villages in the commune. They are responsible for collating nutrition data at the end of each month. Their approach is a variation of house visits and temporary set-up of a treatment site. The government was responsible to train professional health workers to coordinate within the CMAM programme.

Emary (2021) states that the programme was successful in reducing malnutrition and mortality of SAM children. Although this success relates directly to the use of trained $\mathrm{CHVs}$ and commune supervisors, the challenge was to find women $\mathrm{CHVs}$ due to the criteria on literacy. Furthermore, although there was good coordination with the provincial and municipal health departments, there were challenges related to keep in line with the international protocol that all SAM children should first be checked in health centres before going into community treatment. More appropriate tools are needed for CHVs to improve methods for follow-up on referral and discharged cases, improved training on monitoring and evaluation (M\&E). There were also significant stock-outs of RUTF due to national shortages. And it was recommended to have men attending nutrition education sessions.

\footnotetext{
${ }^{14}$ Source for this case study is from a recent presentation by Emary (2021) who works for World Vision International.
} 


\section{Nigeria $^{15}$}

In Nigeria the CMAM information system is based on weekly (SMS) and monthly (paper-based) collection of data that work complementary. The SMS system enables rapid response to stock shortages, while the data from the paper-based system are more compatible with other health service reporting systems (Mezger et al., 2018). The system is implemented by the Nigerian government and supported by UNICEF.

An evaluation report of Oxford Policy Management states that technical aspects of data transmission work well for both the SMS- and paper-based systems, apart from occasional lack of network connectivity that affects the completeness and timeliness of the SMS data (Mezger et al., 2018). Because stakeholders recognise the importance of data for monitoring and developing the CMAM programme, there is high motivation and therefore good potential to improve the system (Mezger et al., 2018). Errors were mainly contributed at the stages of data collection and transmission by health workers. One important point mentioned in the report is the lack of quality assurance at the point of data entry. Furthermore, the user-friendliness of the both the weekly and monthly datasets could be improved, and this would likely stimulate great use of the datasets. See for full recommendations Mezger et al. (2018).

\section{Lessons learned from conflict settings}

\section{Using alternative CMAM approaches}

Violent conflict almost always results in higher levels of malnutrition, particularly in children, due to displacement, disruptions in food supplies, and destroyed crops (Özerdem and Roberts, 2012; IFPRI, 2015; Carroll et al., 2017; Quak, 2018), while affecting vital future agriculture investment (Kimenyi et al., 2014). Hence, it is important to understand how CMAM programmes could work or should be amended in conflict settings. Interventions that mobilise local community participation to improve local capacity building and sustainability of acute malnutrition programmes, such as CMAM, will become particularly important in the coming years as funding for humanitarian food aid continues to decrease globally (Carroll et al., 2017). In the timeframe of this rapid review, it seems that there is far less specific evidence and learning from CMAM programmes in conflict and fragile settings. Most evidence is on nutrition interventions in general in conflict settings (Shah et al., 2021). Some interesting findings from sources on CMAM programmes in Yemen and South Sudan could be found and will be presented in this section.

Laker and Toose (2016) mention some specific challenges for CMAM programmes in South Sudan.

- Capacity for coordination: Fragility and stretched capacity of the South Sudan government means that they are unable to coordinate a system-wide mobilisation. Therefore, the bulk of leadership and service delivery for CMAM programmes relied on UN agencies and NGO partners.

- Gaps in multisectoral resources: CMAM impacts are significantly undermined if adequate food is unavailable and access to health and nutrition services is limited. This

${ }^{15}$ Source for this case study is Mezger et al. (2018) 
means linkages with other programmes are lacking, which often means lower outcomes for higher costs.

- Breakdown in RUTF supply chain: Conflict and fragility often means that local production of RUTF and other ready-to-use supplementary food products is not possible or unpredictable. International agencies such as WFP and UNICEF can step in to import and distribute such food supplies, but that is expensive and stretches already low funding resources, often resulting in problems to deliver these food products to communities.

- Lack of health facilities: CMAM needs access to inpatient and outpatient treatment centres, but during conflicts many of these centres are destroyed or unable to operate within a non-existent or weak health system. The question can be posed the even if health centres are available, malnourished children and pregnant women can actually access them.

- Movement of people: Displaced people are difficult to track and even when they are reached and involved in CMAM movement of people could make ongoing contact with these people in CMAM programmes complicated.

To tackle these challenges, adaptations are needed for CMAM approaches in conflict and fragility contexts (Shah et al., 2021; Kozuki et al., 2020; Laker and Toose, 2016, Al-Dheeb et al., 2018). Most importantly these studies - in some way or another - show that operating outside the health system means that agencies and NGOs that commit to deliver CMAM in conflict settings have to lead, plan, and fund activities themselves. They must be committed to provide and distribute equipment to specific sites (e.g. OTP sites) or through mobile units and deliver capacity building for CHVs without or with limited support from health centres. Laker and Toose (2016) mention that development and implementation of a Rapid Response Mechanism (RRM) within CMAM the CMAM approach in South Sudan resulted in better practice of intervention delivery as it deploys mobile teams of nutrition and health specialists to sites and communities under pressure.

Still, many challenges remain due to high logistical costs and insecurities, which both have been found in South Sudan and Yemen studies (Laker and Toose, 2016; Al-Dheeb et al., 2018). In Yemen, for example, Al-Dheeb et al. (2018) state that the CMAM approach needed to be evolved to meet increased caseload and includes mobile teams, integration with vaccination and community health worker services, and increased geographical coverage. The programme was initially implemented through fixed health facilities only; however, only $60 \%$ of the population had access to health services, a situation which deteriorated further as a result of the conflict; by 2018 only $50 \%$ of health facilities were functional (Al-Dheeb et al., 2018). The Yemen government and partners, therefore, broadened the implementation modalities to improve access and coverage; for example, by integrating the nutrition component into vaccination campaigns and using mobile teams to reach remote areas with no existing fixed health institutions, conflictaffected areas where services have been obstructed, and displacement areas. However, many challenges remained as these improvements and coverage were hampered by low funding for CMAM (particularly prior to the 2017-2018 famine) ${ }^{16}$, import restrictions on supplies, limited

\footnotetext{
${ }^{16}$ While there have been significant increases in funding in 2017, funding remains below necessary levels (the CMAM programme was only $69.7 \%$ funded in 2017 ), which especially effected the MAM component, which could have increased SAM cases due to lack of care for MAM children (AI-Dheeb et al., 2018).
} 
access to increase insecurities, limited numbers of CHVs (with less than $25 \%$ of needed CHVs in place and a limited number of mobile teams) and unpaid health worker salaries, and there are competing priorities in the health system, such as the cholera outbreak, which impacted CMAM programme implementation (Al-Dheeb et al., 2018).

These outcomes seem in line with research that shows that in Yemen the factors related to poor accessibility, poor satisfaction with staff and system, and factors related to treatment and acceptability of OTP services were significantly associated with a high $(55 \%)$ default rate in a SAM programme in Sana'a (Al Amad et al., 2017). This shows that expansion of OTP services and further training OTPs staff on SAM treatment protocols are highly recommended.

Laker and Toose (2016) recommend that more flexibility is needed to enable partners to implement the RRM within CMAM, allowing it where there are no field-level agreements or partnership corporate agreements in place. Furthermore, integration of food-aid mobile teams and nutrition RRM teams would enable partners to implement the full continuum of CMAM, including interventions to prevent malnutrition. Mapping of capacity gaps among partners prior to RRM design would help identify specific areas of intervention, avoiding conflict and duplication of activities.

Some specific actions for adaptations are:

- Commitments from humanitarian agencies and NGOs to attend bi-weekly cluster meetings to coordinate responses and improve information sharing and management (Laker and Toose, 2016).

- Stock RUTF from different sources to create a buffer for times when the main supplies dry up. Supply RUTF to communities less frequently (e.g. monthly instead of weekly) (Laker and Toose, 2016).

- When health centres are operational, supply them well before the raining season starts and roads become inaccessible (Laker and Toose, 2016).

- Budgets need to be predictable and long-term to secure continuity in the absence of a transition to be integrated within health systems (Laker and Toose, 2016).

- Critical actions should be taken to scale-up quality CMAM programmes by focussing on outreach within communities, scaling-up MAM treatment as they are often set aside due to lack of funding (Al-Dheeb et al., 2018).

- Continue with livelihood interventions to help alleviate food insecurity (Al-Dheeb et al., 2018).

$\mathrm{CHVs}$ can fill in some of the gap left by the lack of health centre staff in conflict and fragile settings. They continue screening and treatment and referring the most severe SAM children to health centres or OTPs as long as they have access to RUTF and other supplementary foods. Family MUAC could also help to find cases earlier. The question remains, could low-literate CHVs deliver good outcomes with simplified protocols? In 2016, the International Rescue Committee (IRC), Action Against Hunger, and the London School of Hygiene and Tropical Medicine established a simplified protocol for CMAM (Bailey et al., 2016; Tesfai \& Bailey, 2017). This protocol eliminates the binary distinction between SAM and MAM, and instead offers a unified and simplified protocol for treating both was developed (named ComPAS, Combined Protocol for Acute Malnutrition Study). 
Kozuki et al. (2018) conducted a feasibility study to test the efficiency of adapted or such simplified protocols for CMAM in conflict areas that was conducted in South Sudan's Aweil South County. ${ }^{17}$ For the study $60 \mathrm{CHVs}$ with low literacy were selected and trained to work with simplified methods on screening, treating, and referring malnourished children. For example, they redesigned the MUAC tape, simplified the weight scale and RUTF dosage calculations, simplified the patient register, health records, and SAM treatment algorithms cards, and visualised feeding message flipcharts for caregivers. The aim of the study was to study whether low-literate CHVs can use simplified tools to effectively treat SAM without medical complications, while making referrals of OTPs for children with complications, with no appetite, and when no progress can be measured after four weeks. The study demonstrated that in the context of South Sudan the CHVs were able to treat SAM children in their homes with high accuracy and achieve acceptable recovery rates that meet the Sphere humanitarian minimum standards despite treating children with lower MUAC on admission as compared to the facility. The performance scores were higher among those who received more supervision visits.

This suggests that such a programme working with low-literate CHVs with simplified methods, can successfully be integrated into an integrated Community Case Management (iCCM) protocol within conflict settings. Other findings show the importance of sensitising community leaders and mobilising communities, which was less advanced for this study, with meetings only held at the beginning of the study. Several caregivers expressed distrust of the CHVs and suspected that they were eating RUTF or the general food rations. Therefore, Kozuki et al. (2018) recommend for future programming that community dialogue should be more extensive and should be more frequently conducted. Finally, the study did not show problems with the distribution of RUTFs to communities, but they admit this was probably the result of having a supervisor for two CHVs, which made it less complicated for them to travel and collect RUTF, but on a larger scale this would complicate distribution (Kozuki et al., 2018).

These outcomes are in line with an analysis of data from children recovering from SAM in OTPs and from MAM in targeted SFPs in Chad, Kenya and Yemen (Bailey et al., 2016). It shows that although a simplified protocol can treat SAM children, even with logistical and financial constraints that make one RUTF available for each child a week instead of the recommended two, it is logistically complicated to implement. It also requires the procurement of two different nutritional products and the set-up of two separate programmes (including staff and training needs in the OTPs), in coordination with two separate (usually UN) agencies (Bailey et al., 2016). This binary system often results in the prioritisation of SAM over MAM (Tesfai \& Bailey, 2017), which therefore decreases the availability of treatment, which could result that treatment may not be available to children with MAM until they deteriorate to SAM when resources are limited (Save the Children, 2017; Bailey et al., 2018).

As it can be concluded that combined treatment for SAM and MAM is non-inferior to standard care (Bailey et al., 2020), still further research is needed to understand operational implications. Kozuki et al. (2020) studied the protocols of combined/simplified protocols in CMAM programmes during emergency situations in Niger, Nigeria, Somalia, and South Sudan. They found that while combined/simplified protocols for outpatient management of uncomplicated cases of acute malnutrition are being used in emergency situations in all four countries, there is widespread

\footnotetext{
17 The study was conducted by International Rescue Committee and funded by the Eleanor Crook Foundation. See more in Kozuki et al. (2018).
} 
confusion about protocol terminology and content. This can be traced back to a lack of coherence at the global level (Kozuki et al., 2020). As a result, stakeholders implement programmes based on diverse, overlapping, and inconsistent rationales for modifying the standard protocols to cope with the emergency situation. Kozuki et al. (2020), therefore, conclude that without specific global-level guidance, combined/simplified protocols will continue to be used on an ad hoc basis. As such, standardised guidance based on the available evidence is required to clarify best practices for combined management of SAM and MAM, particularly in emergency contexts (which should be defined) and in situations of limited resources (Kozuki et al., 2020).

Overall, the literature mention that few studies have assessed the cost-effectiveness of nutrition interventions, particularly with regard to treatment of MAM and changes in acute malnutrition protocols (Lenters et al., 2016: Lelijveld et al., 2018).

\section{Case studies}

\section{Somalia ${ }^{18}$}

Action Against Hunger in Somalia adapted their CMAM programme in 2020 due to Covid-19 outbreak. These included modified frequency of follow-up appointments during acute malnutrition treatment; and suspension of screening and outreach by CHVs and scale-up of Family MUAC for early case identification.

The State of Acute Malnutrition website, where this case was mentioned, states that to reduce overcrowding at clinics, Action Against Hunger reduced the frequency of scheduled follow-up visits for SAM children in the programme from weekly to bi-weekly visits, and follow-up for children with MAM was shifted from bi-weekly to monthly appointments. Programme staff increased messaging and sensitisation at the clinics and in the community to resolve confusion. Staff also suggested that increased time between visits might result in sharing of nutrition supplies among other children in the household. Healthcare workers were therefore concerned that children may regress or lose weight as a result of the adaptation. Staff recommended that increased follow-up of children at the community level by CHVs could promote adherence and minimise missed visits and non-response.

Mass screening and surveillance were severely restricted in Somalia under COVID-19 guidance, as such to promote continued early identification of cases, Action Against Hunger implemented Family MUAC in line with official guidance. Mothers and caregivers received training both at clinics and within the community in small groups of 5-10 individuals. It has reportedly reduced the workload of $\mathrm{CHVs}$ within the community, as it is intended to shift primary ownership of screening over to caregivers. The primary challenge in implementing this adaptation was reported to be a limited supply of MUAC tapes, therefore restricting participation at the initial stage.

Lessons learned were that enhanced and strengthened community response and communitybased interventions continued access to services despite fear of coming to facilities. Participants

\footnotetext{
18 The source of this case study is The State of Acute Malnutrition website. Available at: https://acutemalnutrition.org/en/innovations-and-covid19-adaptations-case-studies-somalia
} 
were in favour of continued implementation of Family MUAC after COVID-19 to enhance community-based screening, support caregivers in monitoring their children's health and nutrition status and identify and enroll malnutrition cases early. However, many challenges remain. Adequate essential supplies must be prepositioned to avoid stockouts. International lockdowns also led to disruptions in the supply pipeline. Finally, given the noted increases in lengths of stay and reduced rate of weight gain, programmes operating with increased durations between followup visits must be closely monitored in order to ensure that children are receiving optimal care.

\section{Yemen $^{19}$}

One example of an integrated community programme is the partnership between UNICEF and a local Yemeni NGO, Soul for Development. This was set-up to implement a pilot project for a twoyear, integrated, community-based programme in three out of 23 districts in the southwest city of Ta'izz (208 villages with a combined population of 312,634) from March 2015 to February 2017. The project used the 'triple A' approach, which enables communities to Assess and Analyse the causes of their health and nutrition-related problems and identify and agree 'doable Actions' at the family and community levels to improve health and nutrition status (Sallam et al., 2017).

The entry point to community engagement was the formation of 193 village development committees (VDCs) including 8-10 influential people in the community, including school principals, traditional leaders, sheikhs (tribal leaders) and religious leaders such as imams. A further 15 sub-district development committees were also formed. These committees, together with the respective District Health Office, were involved in the selection of 770 female CHVs, who were trained to provide health and nutrition services in $50 \%$ of the villages. The main roles of these $\mathrm{CHVs}$ were to screen children for acute malnutrition and refer them for treatment, as well as to provide micronutrient powders to children under two years old, iron and folate to pregnant women, and deworming tablets to under $5 \mathrm{~s}$.

The main challenges involved communication and coordination issues, especially with health offices at governorate and district levels. The local NGOs have to deal with multiple and conflicting 14 authorities (the Ministry of Health on the one hand, and the de facto authorities on the other), with both parties imposing contradicting instructions and demands (Sallam et al., 2017). To help with this, a review workshop was organised with NGO partners working in CMAM programmes, including Soul for Development, to inform scaling-up plans, improve volunteer selection, building capacity for health supervisors and mobile health teams, unifying planning and coordination, and an emphasis on education and support to adopt the production of local foodbased meals and recipes. However, a further step is needed to create a multi-sector, communitybased programme that provides both nutrition specific and nutrition sensitive interventions in order to unify provision of SAM and MAM services.

\footnotetext{
19 The source of this case study is Tull (2018) where the study of Sallam et al. (2017) was presented.
} 


\section{Appendix: Logic model used for CMAM evaluations}

https://evaluationreports.unicef.org/GetDocument?fileID=5812

Source: UNICEF (2013)

\section{References}

Aguayo, V.M., Badgaiyan, N., Qadir, S.S., Bugti, A.N., Alam, M.M., Nishtar, N., Galvin, M. (2018). Community management of acute malnutrition (CMAM) programme in Pakistan effectively treats children with uncomplicated severe wasting. Maternal \& Child Nutrition, 14(S4). DOI:10.1111/mcn.12623

Al Amad, M., Al-Eryani, L., Al Serouri A., Khader, Y.S. (2017). Evaluation of outpatient therapeutic programme (OTP) for treatment of severe acute malnutrition in Yemen: a focus on treatment default and its risk factors. Journal of Evaluation in Clinical Practice, 23(6), p.1361-1366. DOI:10.1111/jep.12798

Alé, F.G., Phelan, K.P., Issa, H., Defourny, I., Le Duc, G., Harczi, G., Issaley, K., Sayadi, S., Ousmane, N., Yahaya, I., Myatt, M., Briend, A., Allafort-Duverger, T., Shepherd, S., Blackwell, N. (2016). Mothers screening for malnutrition by mid-upper arm circumference is non-inferior to community health workers: results from a large-scale pragmatic trial in rural Niger. Archives of Public Health, 74(1), p.38. DOI:10.1186/s13690-016-0149-5

Al-Dheeb, N., Ziolkovska, A., Chitekwe, S. (2018). Experiences of implementing CMAM in Yemen and number of deaths averted. Field Article. Available at:

https://www.ennonline.net/attachments/2934/FEX_58_Yemen_p64.pdf (PDF)

Bailey, J., Opondo, C., Lelijveld, N., Marron, B., Onyo, P., Musyoki, E.N., Adongo, S.W., Manary, M., Briend, A., Kerac, M. (2020). A simplified, combined protocol versus standard treatment for acute malnutrition in children 6-59 months (ComPAS trial): A cluster-randomized controlled non-inferiority trial in Kenya and South Sudan. PLOS Medicine.

DOI:10.1371/journal.pmed.1003192

Bailey, J., Lelijveld, N., Marron, B., Onyoo, P., Ho, L.S., Manary, M., Briend, A., Opondo, C., \& Kerac, M. (2018). Combined Protocol for Acute Malnutrition Study (ComPAS) in rural South Sudan and urban Kenya: study protocol for a randomized controlled trial. Trials, 19: 251. DOI:10.1186/s13063-018-2643-2

Bailey, J., Chase, R., Kerac, M., Briend, A., Manary, M., Opondo, C., Gallagher, M., \& Kim, A. (2016). Combined protocol for SAM/MAM treatment: The ComPAS study. Field Exchange, 53. Available at: https://www.ennonline.net/attachments/2544/FEX-53-Web_30112016.pdf (PDF)

Bhutta, Z.A., Das, J.K., Rizvi, A., Gaffey, M.F., Walker, N. Horton, S., Webb, P., Lartey, A. (2013). Evidence-Based Interventions for Improvement of Maternal and Child Nutrition: What Can Be Done and at What Cost? The Lancet Maternal and Child Health series, 382(9890). DOI:10.1016/S0140-6736(13)60996-4 
Blackwell N, Myatt M, Allafort-Duverger T, Balogoun A, Ibrahim A, Briend A. (2015).. Mothers Understand and Can do it (MUAC): a comparison of mothers and community health workers determining mid-upper arm circumference in 103 children aged from 6 months to 5 years. Archives in Public Health, 73(1), p.26. DOl10.1186/s13690-015-0074-z

Bliss, J., Lelijveld, N., Briend, A., Kerac, M., Manary, M., McGrath, M., Weise Prinzo, Z., Shepherd, S., Marie Zagre, N., Woodhead, S., Guerrero, S., \& Mayberry, A. (2018). Use of Mid-Upper Arm Circumference by Novel Community Platforms to Detect, Diagnose, and Treat Severe Acute Malnutrition in Children: A Systematic Review. Global health, science and practice, 6(3), p.552-564. DOI:10.9745/GHSP-D-18-00105

Bliss, J., Golden, K., Bourahla, L., Stoltzfus, R., Pelletier, D. (2018). An emergency cash transfer program promotes weight gain and reduces acute malnutrition risk among children 6-24 months old during a food crisis in Niger. Journal of Global Health, 8(1), p.010410. DOI:10.7189/jogh.08.010410

Briend A, Maire B, Fontaine O, Garenne M. Mid-upper arm circumference and weight-for-height to identify high-risk malnourished under-five children. Maternal Child Nutrition, 8(1), p.130-3. DOI:10.1111/j.1740-8709.2011.00340.x

Carroll, G.J., Lama, S.D., Martinez-Brockman, J.L., Pérez-Escamilla, R. (2017). Evaluation of Nutrition Interventions in Children in Conflict Zones: A Narrative Review. Advances in Nutrition, 8(5), p.770-779. DOI:10.3945/an.117.016121

Chanani, S., Wacksman, J., Deshmukh, D., Pantvaidya, S., Fernandez, A., Jayaraman, A. (2016). M-Health for Improving Screening Accuracy of Acute Malnutrition in a CommunityBased Management of Acute Malnutrition Program in Mumbai Informal Settlements. Food and Nutrition Bulletin, 37(4), p.504-516. DOI:10.1177/0379572116657241

Concern (2015). Concern Worldwide's Learning from 15 years of Community Management of Acute Malnutrition Programming. Available at:

https://admin.concern.net/sites/default/files/media/migrated/concern_worldwides_learning_fr om_15_years_of_community_management_of_acute_malnutrition_programming.pdf?_gl=1* 1Isrilc*_ga*NjU1MDgzNzMuMTYzMzA4MDYzOA..*_ga_RLZ9XCKFP1 *MTYzMzYwNzM3N S4zLjEuMTYzMzYwNzQ2MC42MA (PDF)

Emary, C. (2021). Using Community Volunteers to deliver treatment services for acute malnutrition in Bie, Huambo, Zaire and Kwanza Sul, Angola. Presentation of World Vision. Available at: https://www.wvi.org/sites/default/files/202103/Community\%20Health\%20Workers\%20\%26\%20CMAM\%20\%20WV\%20Angola\%20CMAM\%20Presentation.pdf (PDF)

FANTA (2018). Training Guide for Community-Based Management of Acute Malnutrition (CMAM). Food and Nutrition Technical Assistance III Project (FANTA). Washington, DC: FHI 360/FANTA. Available at: https://reliefweb.int/sites/reliefweb.int/files/resources/CMAMTraining-GUIDE-Sep2018.pdf (PDF)

Goudet, S., Jayaraman, A., Chanani, S., Osrin, D., Devleesschauwer, B., Bogin, B., Madise, N., Griffiths, P. (2018). Cost effectiveness of a community-based prevention and treatment of 
acute malnutrition programme in Mumbai slums, India. PLOS One.

DOI:10.1371/journal.pone.0205688

Grellety, E., Golden, M.H. (2016). Weight-for-height and mid-upper-arm circumference should be used independently to diagnose acute malnutrition: policy implications. BMC Nutrition, 2(10). DOI:10.1186/s40795-016-0049-7

Huybregts, L., Becquey, E., Zongrone, A., Le Port, A., Khassanova, R., Coulibaly, L., Leroy, J., Rawat, R., Ruel, M. (2017). The impact of integrated prevention and treatment on child malnutrition and health: the PROMIS project, a randomized control trial in Burkina Faso and Mali. BMC Public Health, 17, 237 (2017). DOI:10.1186/s12889-017-4146-6

Huybregts, L., Becquay, E., Leroy, J., Ruel, M. (2019). Incorporating prevention into communitybased management of acute malnutrition: Insights from Mali and Burkina Faso. IFPRI blog. Available at: https://www.ifpri.org/blog/incorporating-prevention-community-basedmanagement-acute-malnutrition-insights-mali-and

IFPRI (2015). 2014-2015 Global Food Policy Report. International Food Policy Research Institute, Washington, DC. Available at:

http://ebrary.ifpri.org/cdm/ref/collection/p15738coll2/id/129072

Ireen, S., Raihan, M.J., Choudhury, N., Islam, M.M., Hossain, M.I., Islam, Z., Rahman, S.M.M., Ahmed, T. (2018). Challenges and opportunities of integration of community based Management of Acute Malnutrition into the government health system in Bangladesh: a qualitative study. BMC Health Services Research, 18(1), p.256. DOI:10.1186/s12913-0183087-9.

Kimenyi, M., Adibe, J., Djiré, M., Jirgi, A.J., Kergna, A., Deressa, T.T., Pugliese, J.E., Westbury, A. (2014). The impact of conflict and political instability on agricultural investments in Mali and Nigeria. Africa growth initiative working paper 17. Available at:

https://www.brookings.edu/wp-content/uploads/2016/06/14-07-22-Impact-of-ConflictMaliNigeria_FINAL.pdf (PDF)

Kozuki, N., Seni, M., Sirat, A. et al. (2020). Adapting acute malnutrition treatment protocols in emergency contexts: a qualitative study of national decision-making. Conflict and Health, 14(47). DOI:10.1186/s13031-020-00293-x

Kozuki, N., Ericson, K., Marron, B., Barbera, Y., \& Miller, N. (2017). Integrated Community Case Management in Acute and Protracted Emergencies Case Study for South Sudan May 2017. IRC and UNICEF. Available at:

https://www.rescue.org/sites/default/files/document/1740/ircunicefsouthsudaniccmcasestudy final.pdf

Laker, M., Toose, J. (2016). Nutrition programming in conflict settings: lessons from South Sudan. Field Exchange, 53(2). Available at: https://www.ennonline.net/fex/53/nutritionprogramminginsouthsudan

Lelijveld, N., Bailey, J., Mayberry, A., Trenouth, L., N'Diaye, D.S., Haghparast-Bidgoli, H., \& Puett, C. (2018). The "ComPAS Trial" combined treatment model for acute malnutrition: 
study protocol for the 17 economic evaluation. Trials, 19, 252. DOI:10.1186/s13063- 0182594-7

Lenters, L., Wazny, K., \& Bhutta, Z.A. (2016). Chapter 11: Management of Severe and Moderate Acute Malnutrition in Children. In Black RE, Laxminarayan R, Temmerman M, et al., [editors]. "Reproductive, Maternal, Newborn, and Child Health: Disease Control Priorities, Third Editioin (Volume 2). Washington (DC): The International Bank for Reconstruction and Development / The World Bank; 2016. Available at: https://www.ncbi.nlm.nih.gov/books/NBK361900/

Mezger, C., Tuffrey, V., Umar, Charles, Olisenekwu, G., Namukasa, E. (2018). Community Management of Acute Malnutrition (CMAM) in Nigeria: Performance assessment of the CMAM information system. Oxford Policy Management. Available at: ms2-opm-cmam-datasystems-final-report.pdf (opml.co.uk)

Moramarco S, Amerio G, Chafula Muyaba L, Bonvecchio D, Abramo E, Palombi L, Buonomo E. (2017). Nutritional counseling improves dietary diversity and feeding habits of Zambian malnourished children admitted in Rainbow nutritional programs. Biomedicine \& Prevention issues, 1(93), p.70-76. DOI:10.19252/00000005D.

Moramarco S, Amerio G, Kasengele Chipoma J, Nielsen-Saines K, Palombi L, Buonomo E. (2018). Filling the Gaps for Enhancing the Effectiveness of Community-Based Programs Combining Treatment and Prevention of Child Malnutrition: Results from the Rainbow Project 2015-17 in Zambia. International Journal of Environmental Research and Public Health. 15(9), p.1807. DOI:10.3390/ijerph15091807.

Moramarco S, Amerio G, Gozza, G., Buonomo E., Palombi L. (2019). Enhancing the effectiveness of a community-based management of acute malnutrition programme in Zambia. EnnOnline, Field Exchange issue 60. Available at: https://www.ennonline.net/attachments/3203/FEX-60-Web_final_74-77.pdf (PDF)

Myatt, M., Khara, T., Collins, S. (2006). A review of methods to detect cases of severely malnourished children in the community for their admission into community-based therapeutic care programs. Food and Nutrition Bulletin, 27(3 Suppl):S7-23. DOI:10.1177/15648265060273S302

Özerdem, A., \& Roberts, R. (eds). (2012). Challenging Post-conflict Environments: Sustainable Agriculture. Taylor and Francis.

Park, S.E., Kim, S., Ouma, C., Loha, M., Wierzba, T.F., Beck, N.S. (2012). Community management of acute malnutrition in the developing world. Pediatric Gastroenteology Hepatology and Nutrition, 15(4), p.210-219. DOI:10.5223/pghn.2012.15.4.210

Quak, E. (2018). Food Systems in Protracted Crises: Strengthening Resilience against Shocks and Conflicts. K4D Helpdesk Report 447. Brighton, UK: Institute of Development Studies. Available at: https://www.ids.ac.uk/publications/food-systems-in-protracted-crisesstrengthening-resilience-against-shocks-and-conflicts/ 
Sallam, F.A.K., Albably, K., Zvandaziva, C., \& Singh K. (2017). Community engagement through local leadership: Increasing access to nutrition services in a conflict setting in Yemen. https://www.ennonline.net/nex/9nutserviceaccessyemen

Save the Children (2020). Family MUAC Approach in the Time of COVID-19: Implementation guidance for programme managers. Available at:

https://resourcecentre.savethechildren.net/library/family-muac-approach-time-covid-19implementation-guidance-programme-managers

Save the Children (2017). Nutrition and Mortality Assessment Report Emergency WASH \& Nutrition for conflict affected people in Yemen - Taiz governorate. Available at:

https://reliefweb.int/sites/reliefweb.int/files/resources/Taiz_highlands_7_districts_smart_surv ey_report_march_2017.pdf (PDF)

Shah, S., Padhani, Z.A., Als, D., et al. (2021). Delivering nutrition interventions to women and children in conflict settings: a systematic review. BMJ Global Health. DOI:10.1136/ bmjgh2020-004897

Tesfai, C., Bailey, J. (2017). Breaking down the barriers to treating malnutrition. (18 January 2017) IRIN News. Available at: https://www.irinnews.org/opinion/2017/01/18/breaking-downbarriers-treating-malnutrition

Tull (2018). Simplified Protocol to Treat Severe and Moderate Acute Malnutrition in Yemen, K4D Helpdesk Report. Brighton, UK: Institute of Development Studies. Available at:

https://opendocs.ids.ac.uk/opendocs/handle/20.500.12413/13807

UNICEF (2013). Evaluation of Community Management of Acute Malnutrition: Global Synthesis Report. Washington, DC: UNICEF. Available at:

https://evaluationreports.unicef.org/GetDocument?fileID=5812

UNICEF. 2015. Management of Severe Acute Malnutrition in Children: Working Towards Results at Scale. New York: UNICEF. Available at: https://reliefweb.int/report/world/managementsevere-acute-malnutrition-children-working-towards-results-scale-unicef-2015

UNICEF (2020). Screening of Acute Malnutrition by the Family at community level. Rapid Review. Washington, DC. Available at:

https://www.unicef.org/wca/media/5716/file/Family\%20MUAC.pdf (PDF)

UNICEF, Global Nutrition Cluster, Global Technical Assistance Mechanism for Nutrition (2020). Management of child wasting in the context of COVID-19. Brief, no.1. Washington, DC. Available at: https://www.unicef.org/documents/management-wasting-context-covid-19

USAID (2016). Multi-sectoral nutrition strategy 2014-2015. Technical Guidance Brief on Community-based Management of Acute Malnutrition. Washington, DC: USAID. Available at: https://www.usaid.gov/sites/default/files/documents/1864/CMAM-technical-guidancebrief-508-revFeb2017.pdf (PDF)

WHO, WFP, UNICEF, UN Standing Committee on Nutiriton (2007). Community-based management of severe acute malnutrition. A Joint Statement. Available at: 
https://www.who.int/nutrition/topics/Statement_community_based_man_sev_acute_mal_eng .pdf (PDF)

Wilunda, C., Mumba, F.G., Putoto, G. et al. (2021). Effectiveness of screening and treatment of children with severe acute malnutrition by community health workers in Simiyu region, Tanzania: a quasi-experimental pilot study. Scientific Reports, 11, 2342.

DOI:10.1038/s41598-021-81811-6

World Vision (2020). Management of Wasting in the context of COVID-19: Program Adaptations for CMAM Programs. Health \& Nutrition Technical Brief. Available at:

https://www.wvi.org/sites/default/files/2020-

06/CMAM\%20adaptations\%20for\%20COVID_0.pdf (PDF)

\section{Acknowledgements}

I would like to thank the following experts who voluntarily provided suggestions for relevant literature or other advice to support the preparation of this report. The content of the report does not necessarily reflect the opinions of any of the experts consulted.

- Gudrun Stallkamp, Regional Nutrition Advisor East \& Southern Africa, Deutsche Welthungerhilfe.

- Alex Fenwick, Head of Programmes - Somaliland, Deutsche Welthungerhilfe.

\section{Suggested citation}

Quak, E. (2021). Lessons learned from Community-based Management of Acute Malnutrition (CMAM) programmes that operate in fragile and conflict affected settings. K4D Helpdesk Report no.1048. Brighton, UK: Institute of Development Studies. DOI: 10.19088/K4D.2021.133

\section{About this report}

This report is based on six days of desk-based research. The K4D research helpdesk provides rapid syntheses of a selection of recent relevant literature and international expert thinking in response to specific questions relating to international development. For any enquiries, contact helpdesk@k4d.info.

K4D services are provided by a consortium of leading organisations working in international development, led by the Institute of Development Studies (IDS), with Education Development Trust, Itad, University of Leeds Nuffield Centre for International Health and Development, Liverpool School of Tropical Medicine (LSTM), University of Birmingham International Development Department (IDD) and the University of Manchester Humanitarian and Conflict Response Institute (HCRI).

This report was prepared for the UK Government's Foreign, Commonwealth, and Development Office (FCDO) and its partners in support of pro-poor programmes. It is licensed for noncommercial purposes only. K4D cannot be held responsible for errors or any consequences arising from the use of information contained in this report. Any views and opinions expressed do not necessarily reflect those of FCDO, K4D or any other contributing organisation.

(C) FCDO- Crown copyright 2021.

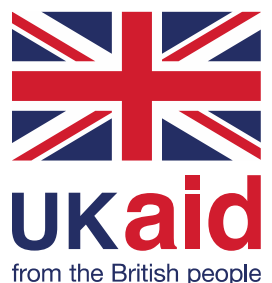

